\title{
Santé publique de précision : Rêve ou réalité?
}

\section{Maureen Dobbins ${ }^{1 *}$, David Buckeridge ${ }^{2}$}

Citation proposée : Dobbins M, Buckeridge D. Santé publique de précision : Rêve ou réalité? Relevé des maladies transmissibles au Canada 2020;46(6):181. https://doi.org/10.14745/ccdr.v46i06a01f

La santé publique de précision (SPP) est définie comme une « nouvelle pratique qui vise à accroitre la précision des processus de prévision et de compréhension des risques de santé publique, ainsi qu'à adapter les traitements à des sous-populations spécifiques et homogènes, souvent par l'utilisation de nouvelles données, technologies et méthodes » (1) [Traduction]. Au Canada, les praticiens de la santé publique commencent à porter une attention particulière à la santé publique de précision et les chercheurs élaborent des méthodes pour sa mise en œuvre. Alors que ces efforts s'accélèrent, il est important de se demander si les avantages prévus pourraient un jour se concrétiser ou si nous sommes à la poursuite d'un rêve irréalisable?

Un peu comme la santé publique fondée sur des données probantes (2), la santé publique de précision (3) a le potentiel de modifier, de façon significative, le processus de prise de décision et d'avoir des incidences positives sur la santé de la population. Ces deux approches sont fondées sur le recours aux meilleures données probantes accessibles pour classifier l'état de santé de la population et pour déterminer les interventions optimales pour une population, compte tenu de son état de santé $(2,4)$. Elles ont toutes deux été rendues possibles par la croissance exponentielle observée au cours de la dernière décennie des capacités relatives aux données et à l'informatique. À titre d'exemple, la plateforme logicielle PopHR intègre un large éventail de données sur la santé de la population qui utilise des connaissances épidémiologiques afin d'orienter la prise de décisions sur les interventions en santé publique (5). Associées à l'utilisation d'un répertoire résumé de données de recherche de haute qualité qui rend possible l'évaluation de l'efficacité des interventions en santé publique (Health Evidence ${ }^{T M}$ ) (6), ces plateformes logicielles pourraient permettre d'atteindre différents avantages associés à la santé publique de précision.

Un projet pilote visant à coder les données de recherche sur les interventions liées à la lutte antigrippale a permis de démontrer qu'il pourrait être possible de créer une base de connaissances codées sur les interventions liées à la lutte antigrippale et que cette base de données pourrait être « lue » par un ordinateur avant d'être jumelée à des données locales pour indiquer quelles sont les interventions qui s'imposent pour répondre à une circonstance particulière. Cela étant dit, la mise en œuvre de cette automatisation a nécessité un capital humain considérable, ce qui représente un obstacle important jusqu'à ce que davantage d'automatisation du processus de codage des preuves se produise. De plus, bien qu'une enquête réalisée en 2019 auprès des professionnels de la santé publique présentait des perceptions très favorables à l'égard du service électronique
Cette oeuvre est mise à la disposition selon les termes de la licence internationale Creative Commons Attribution 4.0

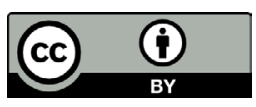

Affiliations

${ }^{1}$ McMaster University, School of Nursing, National Collaborating Centre for Methods and Tools, Hamilton, ON

${ }^{2}$ McGill University, School of Population and Global Health, Montréal, QC

\section{${ }^{\star}$ Correspondance :}

dobbinsm@mcmaster.ca de données probantes pour la santé publique, les décideurs ont également relevé différents enjeux à aborder, comme la prise en considération des valeurs et des ressources communautaires pour être à l'aise avec l'utilisation de cette ressource. Donc, même si l'on a bien des raisons d'être optimistes concernant les avantages que pourrait offrir la santé publique de précision, la réalité est qu'il reste encore beaucoup de notions à assimiler et de travail à faire. Les investissements récemment effectués dans la santé publique et l'informatique placent toutefois le Canada à l'avant-garde de ce nouveau domaine.

\section{Références}

1. Dolley S. Big data's role in precision public health. Front Public Health 2018 Mar 7;6:68. DOI

2. Brownson RC, Fielding JE, Maylahn CM. Evidence-based public health: a fundamental concept for public health practice. Annu Rev Public Health 2009;30(1):175-201. DOI

3. Desmond-Hellmann S. Progress lies in precision. Science 2016 Aug 19;353(6301):731-1. DOI

4. Horton R. Offline: in defence of precision public health. Lancet 2018 Oct 17;392(10157):1504. DOI

5. Shaban-Nejad A, Lavigne M, Okhmatovskaia A, Buckeridge DL. PopHR: a knowledge-based platform to support integration, analysis, and visualization of population health data. Ann N Y Acad Sci 2017 Jan;1387(1):44-53. DOl PubMed

6. Dobbins M, DeCorby K, Robeson P, Husson H, Tirilis D, Greco L. A knowledge management tool for public health: health-evidence.ca. BMC Public Health 2010 Aug 18;10:496. DOI 